\title{
Linux vs. Windows: A Comparison of Application and Platform Innovation Incentives for Open Source and Proprietary Software Platforms
}

Nicholas Economides and Evangelos Katsamakas

\begin{abstract}
The chapter analyzes and compares the investment incentives of platform and application developers for Linux and Windows. We find that the level of investment in applications is larger when the operating system is open source rather than proprietary. The comparison of the levels of investment in the operating systems depends, among others, on reputation effects and the number of developers. The chapter also develops a short case study comparing Windows and Linux and identifies new directions for open source software research.
\end{abstract}

Keywords: Open Source Software, operating systems, technology platforms, Linux, innovation incentives.

JEL Classification: L10, L86, L31.

\subsection{INTRODUCTION}

Open source software is an emerging type of software that may fundamentally affect the business and economic features of the software industry. Linux, an open source operating system, has been the prominent example of the potential of the open source movement, competing against Microsoft Windows, the incumbent operating system. 
This chapter analyzes the incentives to invest in application software and an operating system under two different software ecosystems: one based on an open source operating system, such as Linux, and the other based on a proprietary operating system, such as Microsoft Windows. We build a model extending Economides and Katsamakas (2005) to compare the innovation incentives of application developers and operating system developers for Linux and Windows. In our model, firms and developers invest to improve the quality of the platform or the application and expand the demand by users of these software products. When the operating system is proprietary, the platform provider and the application provider invest only in their own product to maximize their profit. When the operating system is open source, there is no platform provider firm, but the users invest in the platform to maximize their user surplus and their development reputation, which depends on the success of the platform measured by its adoption. This modeling approach is justified by other open source research that conceptualizes the users as developers (Franke and von Hippel 2003, von Hippel 2005).

Another innovation of our model is that it considers the strategic interaction between the platform developers' investment incentives and the application developers' incentives. We show that this interaction is important and should not be ignored in public policy. The existing debate of how innovation is affected by open vs. proprietary platforms (e.g. Lessig 2001) tends to focus only on the innovation incentives of application providers, ignoring the relationship of these incentives with the incentives of the platform provider.

Beyond the analysis of investment incentives, we also present a short case study comparing Windows vs. Linux along three dimensions: the client-side, the server-side and the interaction between the client-side and the server-side. We emphasize that the comparison between Windows and Linux is an issue of comparing two competing software ecosystems, not just two products. The existing Windows ecosystem of the operating system, applications, application developers and service providers is competing against an emerging ecosystem centered on the Linux operating system. The short case study enables us to identify directions for future research on open source software.

The main findings of our analysis are the following. First, the level of investment in the application is larger when the operating system is open source rather than proprietary, when the two operating systems are of equal quality. Second, the level of investment in the operating system depends on a number of factors such as the strength of the reputation effects for the developers of the open source operating system, the ratio of developers within the total user population of the open source operating system, the level of investment in the applications within each ecosystem and the cost of adopting the open source operating system. An increase in one the first two factors leads to a relative increase in the investment 
in the open source operating system, while an increase in the fourth factor leads to a relative increase in the investment in the proprietary operating system.

The chapter has the following structure. Section 10.2 reviews the related literature. Section 10.3 discusses the case study of Windows vs. Linux and identifies some new research directions. Section 10.4 develops the model and analyzes the innovation incentives in the two alternative software ecosystems, the open source and the proprietary one. Section 10.5 concludes the chapter.

\subsection{RELATED LITERATURE}

The literature on economics of open source focuses mainly on the individual incentives to participate in open source projects, the incentives of firms to adopt open source initiatives, the business models of firms operating within the open source landscape and the competitive implications of open source software (Lerner and Tirole 2004, Chapter 2 of this book). ${ }^{1}$ Johnson (2002) models the contribution to an open source project as a problem of private provision of a public good and analyzes the effect of increasing the number of developers. Lerner and Tirole $(2001,2002)$ discuss the incentives of individual programmers and software firms to participate in open source projects. They argue that programmers are motivated by "peer recognition" and delayed career benefits such as being hired by a software firm, or getting access to funding for future software ventures. Mustonen (2003) proposes a model in which the participation of programmers in open source projects is endogenous and shows that a low implementation cost of an open source application is crucial for its survival when it competes with a proprietary application. Casadesus-Masanell and Ghemawat (2003) study a dynamic setting of competition between Windows and Linux. Bitzer (2004) analyzes why some software firms support Linux depending on the heterogeneity between Linux and the firms' commercial products. Economides and Katsamakas (2005) analyze the strategic differences between a proprietary and an open source technology platform and their competition. Mustonen (2005) analyzes when a proprietary software firm may support the development of substitute open source software. Comino and Manenti (2005) assume informed and uninformed users about the existence of open source applications, and study the welfare implications of public policies supporting open source software.

Perhaps the closest paper to this one is that of Bitzer and Schröder (2003), which also analyzes the innovation performance of open source and proprietary

\footnotetext{
' DiBonna and Ockman (1999), Raymond (2001) and Fink (2003) provide good practitioner overviews of open source software.
} 
software development, see also Chapter 12. It shows that competition between open source and proprietary products leads to an increase in the level of technology of both products, as a result of increased investment. The focus of Bitzer and Schröder (2003) is on the effect of competition on innovation, while the focus of our chapter is on a direct comparison of the innovation in the two alternative software ecosystems, which consist of application and operating system developers.

\subsection{CASE STUDY: LINUX VS. WINDOWS}

This case study of Linux vs. Windows distinguishes between the operating systems market for end users - of a desktop operating system (client) - and the market for server operating systems (server). The most interesting battle today is at the server-side.

\subsubsection{Client-side}

Some studies suggest that the market-share of Linux at the client-side is around $3 \%$ and some expect it to reach $7 \%$ by $2007 .^{2}$ This slow growth can be attributed to lack of ease of use, small variety of applications and problems with drivers that enable users to connect other devices to their computing systems. Linux has been mostly an operating system for power-users who have Unix-like skills, but this may change since the open source community is developing several friendly user interfaces such as KDE.

Switching costs from the dominant Windows operating system make it difficult for the Linux market-share to grow fast. Much depends on the relative availability of applications for Windows vs. Linux and the switching costs from the Windows ecosystem to the Linux ecosystem. There are many open source applications under development, and the open source community has recognized the strategic importance of making their applications similar to the Windows applications to lower user switching costs.

Many open source applications (such as OpenOffice, the Mozilla Firefox browser etc.) are also compatible with Windows. Although these applications increase the recognition of the open source community, they strengthen the Windows ecosystem and therefore may hurt Linux in its competition with Windows in the short term. However, the existence of these open source applications may reduce the switching cost to Linux in the long term. At the same time, there are many proprietary applications that are offered over the Linux operating system. Therefore, we do not see a pure open source ecosystem competing with a pure

\footnotetext{
${ }^{2}$ See http://www.idc.com
} 
proprietary ecosystem, but two ecosystems both based on a mix of open source and proprietary applications. Understanding the strategic implications of these mixed ecosystems is an interesting question for future research.

\subsubsection{Server-side}

At the server-side, IDC predicts double-digit growth of Linux adoption ${ }^{3}$ and that Linux server shipments will reach $25.7 \%$ of total shipments in 2008. Linux is "becoming mainstream" and the Linux-based packaged software market is expected to exceed $\$ 14$ million by $2008 .{ }^{4}$

The total cost of ownership (TCO) of Linux may be higher presently. ${ }^{5}$ The migration from Windows environments to Linux is more costly than the migration from Unix to Linux, since Linux is a Unix-like operating system. Therefore a significant switching cost is protecting Windows. Both Windows and Linux are gaining market share at the expense of proprietary Unix systems (including the Sun versions of Unix), which tend to be closed and expensive running on expensive hardware.

The Linux ecosystem is developing fast in terms of number, variety and quality of applications and availability of support and other complementary services. However, it is expected that firms that offer competing proprietary solutions will respond in a variety of ways, including the reduction of prices and higher investment in their products. For example, Microsoft seems committed to reduce the security issues faced by Windows. Uncertainty about potential litigation risks due to unclear property rights and confusing open source licenses also hurts Linux.

Sponsoring of Linux by big IT companies such as IBM and HP is affecting positively Linux because it affects the expectations of customers about the prospects of the platform. These firms are sponsoring Linux by developing or porting their enterprise applications to Linux (such as IBM Websphere), participating actively in open source projects and initiatives and sometimes leading open source projects, announcing publicly their support and their positive expectations about Linux. Sponsoring of Linux by European and Asian governments also strengthens Linux.

Security problems and risks are hurting Windows. An independent study has shown that Linux kernel has 0.17 security flaws per 1,000 lines of code, compared to average $10-20$ flaws of proprietary software. ${ }^{6}$

\footnotetext{
3 eWeek reports "IDC sees double digit growth continuing for Linux", 8 December 2004 at http://www.eweek.com/article2/0,1759,1737068,00.asp.

${ }^{4}$ See http://www.idc.com.

5 See "Yankee independently pits Windows TCO vs. Linux TCO", Microsoft Watch, 24 March 2004, http:/www.microsoft-watch.com/article2/0,1995,1553620,00.asp

6 See "Linux kernel review shows far fewer flaws", eWeek, 14 December 2004, at http://www.eweek.com/ article2/0.1759,1741077,00.asp.
} 
Firms have the option to compile their own for free Linux distribution, by bundling the free Linux kernel with select utilities, but there are several competing commercial Linux distributions (such as Red Hat, SuSe etc.). Therefore there is competition within the Linux platform itself, besides the competition between Linux and Windows. There is a need for more research to analyze that competition and understand the strategies of competing Linux distributors. There is an ongoing process of standardization ${ }^{7}$ across the different distributions, ensuring that a Linux application can run on all distributions. However, it is uncertain how successful this process will be in the future and whether the risk of "forking" to incompatible distributions will materialize. The risk of "forking" is affecting negatively the expectations of potential adopters and the costs of application developers for Linux, who may need to develop different versions of their applications for different Linux distributions.

The existing literature tends to focus on the development side and not on the demand side. It is unclear how and where firms choose to adopt Linux and open source applications. This may depend on the existing IT infrastructure, capabilities and employee IT skills of a firm, the size and the industry of the firm. Only firms with substantial IT capabilities and resources may switch early. Most firms are likely to have a mix of Linux and Windows infrastructure evolving over time. For example, Linux tends to become dominant in Web serving applications, combined with Apache, the dominant Web server application that is open source. ${ }^{8}$ Prominent Internet companies such as Google and Amazon rely heavily on Linux. There is a need for both empirical and analytical work on the conditions under which firms adopt Linux and open source, and how this adoption behavior affects the competition between the two ecosystems.

\subsubsection{Interaction of Server-Side and Client-Side}

There is also a significant relationship between the client/desktop-side and the server-side within an enterprise IT infrastructure. Since Microsoft is expected to have dominance at the client operating systems market (and many applications) for a long time this may give it an advantage at the server market too. Alternatively, as Linux increases its presence at the server market, this may encourage its adoption at the client market as well. The European Union court decision, requiring Microsoft to publish some proprietary protocols that define the interaction of clients and servers, may make it easier to introduce Linux clients in networks that depend on Windows servers. ${ }^{9}$

\footnotetext{
${ }^{7}$ Linux Standards Base (LSB), see http://www.linuxbase.org.

${ }^{8}$ Apache's market-share is larger than 68\%. Microsoft's IIS market-share is around $20 \%$ and the difference between the two is increasing over time; see "Web server survey", Netcraft, January 2005, at http://news. netcraft.com/archives/2005/01/01/january_2005_web_server_survey.html

9 See "Microsoft's secret codes", Wall Street Journal, 22 December 2004.
} 


\subsection{THE MODEL}

This section provides a brief overview of the technology platforms model of Economides and Katsamakas (2005) and extends that model to analyze the investment incentives of different platforms.

The model consists of two software ecosystems, the first based on a proprietary platform and the second based on an open source platform.

The software ecosystem based on the proprietary platform consists of one platform firm selling the platform $\mathrm{A}_{0}$ (operating system), and an independent application developer selling $B_{1}$ (application software). The platform firm sells the platform to the users at a price $p_{0}$. The independent application provider sells the application to the users at a price $p_{1}$. The application provider pays also an access fee $s$ to the platform firm. The fee $s$ is set by the platform firm and it can be negative when effectively the operating system firm subsidizes the application developer. The demand function of the platform $\mathrm{A}_{0}$ is $q_{0}=$ $a_{0}-b_{0} p_{0}-d p_{1}$, and the demand of the application $\mathrm{B}_{1}$ is $q_{1}=a_{1}-b_{1} p_{1}-d p_{0}$, where parameter $d$ measures the complementarity between the platform and the application. We assume that $b_{1}, b_{0}>d$, i.e. the own-price effect of each product dominates the cross-price effect. The profit function of the platform firm is $\pi_{0}=p_{0} q_{0}+s q_{1}$, equal to the platform profit from users plus the platform profit from the application access fees. The profit function of the application provider is $\pi_{1}=\left(p_{1}-s\right) q_{1}$. The marginal cost of production is zero for both the platform and the application, because both are software products easy to reproduce. The firms set prices in a two-stage game. In stage one, the platform sets the access fee $s$ paid by the application provider. In stage two, the platform and the application provider set the user prices $p_{1}, p_{0}$ simultaneously.

The software ecosystem based on an open source platform consists of usersdevelopers that help develop the open source platform $\mathrm{A}_{0}^{\mathrm{O}}$, which is adopted by users for free, and an independent application provider firm that develops and sells the application $\mathrm{B}_{1}^{\mathrm{O}}$ at price $p_{1}^{\mathrm{O}}$. The demand for the platform $\mathrm{A}_{0}^{\mathrm{O}}$ is $q_{0}^{\mathrm{O}}=$ $a_{0}^{\mathrm{O}}-b_{0} e_{\mathrm{u}}-d p_{1}^{\mathrm{O}}$, and the demand for the application $\mathrm{B}_{1}^{\mathrm{O}}$ is $q_{1}^{\mathrm{O}}=a_{1}^{\mathrm{O}}-b_{1} p_{1}^{\mathrm{O}}-d c_{\mathrm{u}}$. Under open source, the profit function of the operating system is zero and the profit function of the application provider is $\pi_{1}^{\mathrm{O}}=p_{1}^{\mathrm{O}} q_{1}^{\mathrm{O}}$. We also assume that open source platform users may face an additional exogenous adoption $\operatorname{cost} c_{\mathrm{u}} \cdot{ }^{10}$

At equilibrium, when the operating system is proprietary, the operating system and application profits excluding investment costs are

$$
\pi_{0}=\frac{a_{1}^{2}\left(2 b_{0} b_{1}+d^{2}\right)+\left(8 b_{0} b_{1}+d^{2}\right)\left(a_{0}^{2} b_{1}^{2}-2 a_{0} a_{1} b_{1} d\right)}{4 b_{1}\left(b_{0} b_{1}-d^{2}\right)\left(8 b_{0} b_{1}+d^{2}\right)}, \quad \pi_{1}=\frac{a_{1}^{2}\left(2 b_{0} b_{1}+d^{2}\right)^{2}}{b\left(8 b_{0} b_{1}+d^{2}\right)^{2}}
$$

\footnotetext{
${ }^{10}$ As noted in the case study (Section 10.3), there is evidence that the TCO of Linux is possibly higher than the TCO of Windows, at least for now.
} 
respectively. Alternatively, when the operating system is open source, equilibrium profits are

$$
\pi_{0}^{\mathrm{O}}=0, \quad \pi_{1}^{\mathrm{O}}=\frac{a_{1}^{\mathrm{O}^{2}} b_{0}+a_{0}^{\mathrm{O}^{2}} b_{1}-2 a_{0}^{\mathrm{O}} a_{1}^{\mathrm{O} d}}{4\left(b_{0} b_{1}-d^{2}\right)}
$$

and the combined consumer surplus from the operating systems and applications markets is

$$
C S^{\mathrm{O}}=\frac{a_{1}^{\mathrm{O}^{2}}\left(b_{0} b_{1}+3 d^{2}\right)-8 d a_{0}^{\mathrm{O}} a_{1}^{\mathrm{O}} b_{1}+4 a_{0}^{\mathrm{O}^{2}} b_{1}^{2}}{8 b_{1}\left(b_{0} b_{1}-d^{2}\right)}
$$

The following section extends this basic framework by introducing new modeling features and assumptions that enable us to analyze and compare the innovation incentives in the two ecosystems.

\subsubsection{Innovation Incentives}

Before the operating systems and the applications are offered to users as defined above, the software firms and individual developers may invest in increasing $a_{i}$ (effectively increasing the quality of the software product), expanding the demand of the operating system and the applications. We show that the incentives to invest differ across operating systems, and we characterize the relative strength of these incentives.

When the operating system is proprietary, we assume that (a) each firm invests in its product to maximize its profit; and (b) neither the users nor the application providers invest in the platform because the platform is closed. For example, the quality of Microsoft Windows depends almost exclusively on Microsoft's investment in this operating system.

When the operating system is open source, we assume that there are $D$ users that are developers, and define the proportion of such users as $\delta=\frac{D}{q_{0}^{0}}<1$. The developers invest in the open source platform so that that they maximize the user/consumer surplus $\mathrm{CS}^{\mathrm{O}}$ from the open source platform plus their reputation value $R\left(q_{0}^{\mathrm{O}}\right)$, where $\mathrm{R}($.$) is an increasing function of the adoption of the open$ source platform. The intuition is that a high adoption of the open platform is a signal of success and all the developers that participated expect some future benefits from that (e.g., future job opportunities). Indeed the development of Linux depends crucially on investment of time and effort of individual developers who also use Linux as an operating system to run the applications they use (Raymond 2001). We assume that the application provider invests in the application, and they may invest on the platform too. 
The game now has an investment stage that precedes the two pricing stages described at the beginning of Section 10.4. We assume that the investment has a fixed cost reflecting the high fixed cost and low variable cost structure of software development. We assume that by investing in a product (operating system or application), the sponsor is able to push outwards (increase) the demand for the product, here measured by the parameter $a_{i}$. The firm incurs a fixed cost indicated by the increasing and convex investment function $C\left(a_{i}\right)$.

Assuming concavity of the profit functions, optimal choice of investment for profit maximization when the operating system is proprietary requires

Operating System:

$$
\frac{\partial \pi_{0}}{\partial a_{0}}=\frac{\partial C\left(a_{0}\right)}{\partial a_{0}} \Rightarrow \frac{a_{0} b_{1}-a_{1} d}{2\left(b_{0} b_{1}-d^{2}\right)}=\frac{\partial C\left(a_{0}\right)}{\partial a_{0}},
$$

Application:

$$
\frac{\partial \pi_{1}}{\partial a_{1}}=\frac{\partial C\left(a_{1}\right)}{\partial a_{1}} \Rightarrow \frac{2 a_{1}\left(2 b_{0} b_{1}+d^{2}\right)^{2}}{b_{1}\left(8 b_{0} b_{1}+d^{2}\right)^{2}}=\frac{\partial C\left(a_{1}\right)}{\partial a_{1}} .
$$

The conditions for developer utility maximization and application firm profit maximization when the operating system is open source are

Operating System:

$$
\delta \frac{\partial C S^{\mathrm{O}}}{\partial a_{0}^{\mathrm{O}}}+\delta \frac{\partial R\left(q_{0}^{\mathrm{O}}\right)}{\partial a_{0}^{\mathrm{O}}}=\frac{\partial C\left(a_{0}^{\mathrm{o}}\right)}{\partial a_{0}^{\mathrm{O}}} \Rightarrow \frac{a_{0}^{\mathrm{O}} b_{1}-a_{1}^{\mathrm{O}} d}{b_{0} b_{1}-d^{2}}+\frac{\partial R\left(q_{0}^{\mathrm{O}}\right)}{\partial a_{0}^{\mathrm{O}}}=\frac{1}{\delta} \frac{\partial C\left(a_{0}^{\mathrm{O}}\right)}{\partial a_{0}^{\mathrm{O}}},
$$

Application:

$$
\frac{\partial \pi_{1}}{\partial a_{1}^{\mathrm{O}}}=\frac{\partial C\left(a_{1}^{\mathrm{O}}\right)}{\partial a_{1}^{\mathrm{O}}} \Rightarrow \frac{a_{1}^{\mathrm{O}}}{2 b_{1}}=\frac{\partial C\left(a_{1}^{\mathrm{O}}\right)}{\partial a_{1}^{\mathrm{O}}} .
$$

We can now prove the following proposition comparing investment incentives in operating systems and applications in the two ecosystems, as described by Equations (10.1)-(10.4).

Proposition 1 Provided that the cost function for software development is the same for open source and proprietary developers and that the open source operating system is offered for free, investment in the application is higher when the operating system is open source, i.e. $a_{1}<a_{1}^{o}$. Investment in the open source operating system is larger than investment in the proprietary operating system if there are strong reputation effects from participation in open source development, and/or a significant part of the open source users are developers and the difference in application investments, $a_{1}^{0}-a_{1}$, is not large. Conversely, investment in a proprietary operating system is smaller when there are weak reputation effects from participation in open source development, and/or only 
a small part of the open source users are developers and the difference in application investment levels, $a_{1}^{\mathrm{O}}-a_{1}$, is large.

Proof: First, compare the maximization conditions of the applications, Equations (10.2) and (10.4). Since $\frac{2 a_{1}\left(2 b_{0} b_{1}+d^{2}\right)^{2}}{b_{1}\left(8 b_{0} b_{1}+d^{2}\right)^{2}}<\frac{a_{1}}{2 b_{1}}$ for all $a_{1}$, and the cost function is convex in $a_{1}$, it follows that $a_{1}<a_{1}^{0}$. Now compare the maximization conditions of the operating systems. Because the cost function is convex, the investment in the open source operating system is larger if and only if

$$
a_{0} b_{1}\left(\delta-\frac{1}{2}\right)+d\left(\frac{a_{1}}{2}-\delta a_{1}^{\mathrm{o}}\right)+\delta\left(b_{0} b_{1}-d^{2}\right) \frac{\partial R}{\partial a_{0}}>0
$$

First, assume that the marginal value of reputation is zero for the open source developers. Then if $\delta \leq \frac{1}{2}$ and $\delta a_{1}^{0} \geq \frac{a_{1}}{2}$, since $b_{0} b_{1}-d^{2}>0$, it follows that Equation (10.5) is false, therefore $a_{0}^{\mathrm{O}}<a_{0}$. If $\delta>\frac{1}{2}$ and $a_{1}^{\mathrm{O}}-a_{1}$ is not large, i.e. if $\delta a_{1}^{\mathrm{O}}<\frac{a_{1}}{2}$ then Equation (10.5) is true, and therefore $a_{0}<a_{0}^{\mathrm{O}}$. Alternatively, since the marginal value of reputation is positive, a strong enough reputation effect such that $a_{0} b_{1}\left(\delta-\frac{1}{2}\right)+d\left(\frac{a_{1}}{2}-\delta a_{1}^{\mathrm{o}}\right)<\delta\left(b_{0} b_{1}-d^{2}\right) \frac{\partial R}{\partial a_{0}}$ is sufficient to fulfill Equation (10.5) and result in $a_{0}<a_{0}^{\mathrm{O}}$.

The proposition suggests that the investment in the application is stronger when the operating system is open source. However, the comparison of investment levels in the operating system is ambiguous. When there are strong reputation effects from participation in the development of the open source operating system, or a significant proportion of the users have also development skills, then the investment in the open source operating system is also larger than the investment of a firm controlling a proprietary operating system. The proposition captures both the reputation benefits and the usage benefits of participants in widely adopted open source projects.

The application provider for the open source operating system invests more than the application provider for the proprietary operating system because the first has a larger marginal profit for all levels of investment. This is because the open source operating system is adopted by users for free, enabling the application provider to set a larger price and capture a larger profit than the application provider for the proprietary operating system.

The level of investment in the applications affects the level of investment in the operating system because of the complementarity between the application and the operating system. In particular, when the level of investment in the application increases, the marginal benefit of investing in the operating system decreases, as shown in Equations (10.1) and (10.3).

Exogenous factors that reduce the adoption of the open source operating system, such as a large adoption cost $c_{\mathrm{u}}$, decrease the incentives of individual 
developers to invest in the open source operating system. Therefore, an independent application provider may subsidize the adoption of the open source platform not only to increase the sales of its application, but also to increase the incentives of developers to invest in the operating system.

\subsection{CONCLUSION}

We developed a model to compare the incentives to invest in the operating system and its applications both under open source and proprietary operating systems. We found that the comparison of the levels of investment in the operating systems is ambiguous, but the investment in applications is stronger when the operating system is open source.

These results were developed under the assumption that the productivity of investment is the same for a proprietary and an open source system. This is an issue where there is significant disagreement, and both sides (for profit software companies and open source developers) claim that their productivity is higher. This clearly is an open research issue, but the purposes of this chapter - a change of the assumption of the equal productivity of investment in both environments will tend to change our result in favor of the ecosystem that is assumed to have higher productivity. For example, if it is assumed that the productivity of the open source development is larger than the productivity of proprietary software development, then $\frac{\partial C^{\mathrm{O}}\left(a_{0}\right)}{\partial a_{0}}<\frac{\partial C\left(a_{0}\right)}{\partial a_{0}}$ for all $a_{0}$ and, everything else being equal, the relative investment in the open source operating system increases; if it is assumed that productivity of proprietary software development is higher, the relative investment in the proprietary operating system increases.

An interesting extension of the current model would be the analysis of the innovation incentives in a competitive setting and the dynamics of innovation.

We also developed a short case study of Linux vs. Windows. The discussion distinguished between issues that affect the competition at the client-side, the server-side and the interaction between the client-side and the sever-side. Besides the competition between Linux and Windows, there is a need for more research to analyze and understand the strategies of competing Linux distributors. Also more research is needed in understanding the switching costs and the open source adoption strategies of enterprises. Another important area is the interaction between client-side and server-side software products and how this interaction affects the competition in the software industry and the success of open source.

\section{ACKNOWLEDGMENTS}

We thank the editors Jürgen Bitzer and Philipp J.H. Schröder for useful comments and suggestions. 


\section{REFERENCES}

Bitzer, J., 2004. Commercial vs. open source software: The role of product heterogeneity in competition. Economic Systems, 28(4), 369-381.

Bitzer, J. and P. J. H. Schröder, 2003. Competition and Innovation in a Technology Setting Software Duopoly. DIW Discussion Paper No. 363.

Casadesus-Masanell, R. and P. Ghemawat, 2003. Dynamic Mixed Duopoly: A Model Motivated by Linux vs. Windows. Harvard Business School Working Paper 04-012.

Comino S. and F. Manenti, 2005. Government policies supporting open source software for the mass market. Review of Industrial Organization, 26(2), 217-240.

DiBonna, C. and S. Ockman, 1999. Open Sources: Voices from the Open Source Revolution. O'Reilly \& Associates, Sebastopol, USA.

Economides, N. and E. Katsamakas, 2006. Two-sided competition of proprietary vs. open-source technology platforms. Management Science, forthcoming.

Fink, M., 2003. The Business and Economics of Linwx and Open Source. Prentice Hall, Upper Saddle River, USA.

Franke, N. and E. von Hippel, 2003. Satisfying heterogeneous user needs via innovation toolkits: The case of Apache security software. Research Policy, 32(7), 1199-1215.

Johnson, J. P., 2002. Open source software: Private provision of a public good. Journal of Economics \& Management Strategy, 11(4), 637-662.

Lemer, J. and J. Tirole, 2001. The open source movement: Key research questions. European Economic Review, 45(4-6), 819-826.

Lerner, J. and J. Tirole, 2002. Some simple economics of open source. Journal of Industrial Economics, 50(2), 197-234.

Lerner, J. and J. Tirole, 2004. The Economics of Technology Sharing: Open Source and Beyond. NBER Working Paper No. 10956.

Lessig, L., 2001. The Future of Ideas. Random House, New York, USA.

Mustonen, M., 2003. Copyleft - the economics of Linux and other open source software. Information Economics and Policy, 15(1), 99-121.

Mustonen, M., 2005. When does a firm support substitute open source programming. Journal of Economics and Management Strategy, 14(1), 121-139.

Raymond, E. S., 2001. The Cathedral and the Bazaar: Musings on Linux and Open Source by an Accidental Revolutionary. O'Reilly \& Associates, Sebastopol, USA.

von Hippel, E., 2005. Democratizing Innovation. MIT Press, Cambridge, USA. 